\title{
Prevalence and Determinants of Depression Among Traumatic Spinal Cord Injured Patients Attending Ibn-Al-Quff Hospital, Baghdad, Iraq
}

Shalan Joodah Rhemah Al Abbudi*, Khalida Ibraheem Ezzat, Ali Abdelilah Zebala, Delkhwaz Jameel Hamdy, Mohammad Shalan Joda AlBeedany and Mohammed Shalal Farhan

Deptartment of Medicine, Al-Imamein Al-Kadhimein Medical City, Baghdad, Iraq

\begin{abstract}
Objective: To identify the prevalence and potential risk factors of depression among spinal cord injured inpatients, and assessment of the severity of depression.

Methods: A cross-sectional study conducted at Ibn Al Quff Hospital for spinal cord injury rehabilitation. All inpatients with traumatic spinal cord injury were recruited excluding severely injured and those injured due to congenital and medical causes. Socio-demographic variables, spinal cord injury characteristics and comorbidity were compiled. Self-Reporting Questioner (SRQ-20) was used to identify mental symptoms. DSM-IV criteria for depression and Hamilton-17 Scale, for assessment of severity of depression were used.

Results: A total of 274 spinal cord injured inpatients were approached; $93 \%$ responded; paraplegics $75.7 \%$ and tetraplegics $24.3 \%$. Violence was the major cause of injury. Seventy four percent $(74.1 \%)$ had depression; $44 \%$ of them had severe and very severe depression. Depression was significantly associated with age $(P=0.001)$, gender $(P=0.001)$, education level $(P=0.038)$, occupation $(P=0.003)$; smoking habit $(P=0.035)$, duration of injury $(P=0.003)$, times of admission $(P=0.000)$, and comorbidity $(P=0.18)$

Conclusion: Prevalence of depression is high and frequent among spinal cord injured patients. Demographic and spinal cord injury variables are significantly associated with depression and are the most important determinants of depression.
\end{abstract}

Keywords: Depression; Spinal cord injury; DSM-IV criteria; Psychological disorders

Abbreviations: SRQ: Self-Reporting Questionnaires; DSM-IV: Diagnostic and Statistical Manual of Mental Disorders-IV; SCI: Spinal Cord Injury; MDD: Major Depressive Disorder; WHO: World Health Organization; HAM-D: Hamilton Scale for Severity of Depression

\section{Introduction}

Spinal cord injury (SCI) is a devastating condition causing profound life changes for millions of people around the world [1]. Over $80 \%$ of traumatic SCI are male, with an average age at injury s of about 40 years and most frequent causes of injuries include motor vehicle accidents, violence, falls, and recreational accidents [2]. SCI typically causes paralysis and permanent disability. Despite costly and aggressive rehabilitative options, injuries to the spinal cord remain permanent and create lifelong challenges for survivors [1]. SCI results in diminished mobility, greatly reduced functional independence, and difficulties with socialization and employment [3]. Exposure to life-threatening conditions or severe mental stress may lead to various psychological reactions including depression. One of the deleterious stresses is that experienced during war. Veterans encumbered with physical disabilities are more prone to depression, among other psychological disorders [4]. During the Iraq-Iran war, many young soldiers and paramilitary troops sustained physical disabilities which were compounded by psychological conflicts [5]. The impact of SCI on psychological status has been variously debated. Several studies have suggested that $\mathrm{SCI}$ is associated with raised risks of psychological problems. Negative psychological states have been found in 30-40\% percent of patients with SCI [6-8]. The Diagnostic and Statistical Manual of Mental DisordersIV (DSM-IV) [9] defines many disorders including MDD on the basis of the presence of a minimum number of symptoms or features from a list $[10,11]$. Depression is the most common psychological issue associated with SCI [4], reportedly affecting approximately $30 \%$ of patients, and is generally characterized by depressed mood and diminished pleasure over a two-week span accompanied by issues including energy loss, concentration difficulties, and sleep or appetite disturbances [9]. There is a large body of literature documenting the high prevalence of depression, psychological distress, and psychological morbidity after spinal cord injury (SCI) [12-14]. In a recent study of community-residing people with traumatic SCI, the rate of probable major depression was found to be 3 times that of the general population [12-15].

In Iraq, Ibn Al-Quff hospital for spinal cord injuries was opened on October 1982 after increased incidence of spinal cord injuries at beginning of Iraq-Iran war $[16,17]$. More than four thousand spinal cord injured (SCI) patients had been rehabilitated in Iraq during the last three decades at Ibn Al-Quff hospital spinal cord injury rehabilitation [18]. About $84.8 \%$ of spinal cord injured persons were paraplegic, and about $15.2 \%$ were quadriplegic persons, $90.5 \%$ male and $9.5 \%$ were female. The causes of SCIs, are approximately $50 \%$ for high velocity missiles, $18 \%$ road traffic accidents, $16 \%$ fall from height, $6 \%$ stab wound, and $10 \%$ for others. A total of 1768 spinal cord injured persons were admitted to the Ibn AL-Quff hospital during 2003-2010 [19].

*Corresponding author: Shalan JR Al-Abbudi, Consultant Psychiatrist, Section of Psychiatry, Department of Medicine, Al-Imamein Al-Kadhimein Medical City, Baghdad, Iraq, Tel: +9647810705221; E-mail: shalanjoodah@yahoo.com

Received: August 10, 2017; Accepted: September 25, 2017; Published: September 30, 2017

Citation: Al Abbudi SJR, Ezzat KI, Zebala AA, Hamdy DJ, Al-Beedany MSJ et al. (2017) Prevalence and Determinants of Depression Among Traumatic Spinal Cord Injured Patients Attending Ibn-Al-Quff Hospital, Baghdad, Iraq. J Psychiatry 20: 428. doi:10.4172/2378-5756.1000428

Copyright: ( 2017 Al Abbudi SJR, et al. This is an open-access article distributed under the terms of the Creative Commons Attribution License, which permits unrestricted use, distribution, and reproduction in any medium, provided the original author and source are credited 
Citation: Al Abbudi SJR, Ezzat KI, Zebala AA, Hamdy DJ, Al-Beedany MSJ, et al. (2017) Prevalence and Determinants of Depression Among Traumatic Spinal Cord Injured Patients Attending Ibn-Al-Quff Hospital, Baghdad, Iraq. J Psychiatry 20: 428. doi:10.4172/23785756.1000428

Page 2 of 7

\section{Patients and Methods}

\section{Design and setting}

This is a cross-sectional study with analytic component. It was conducted in Ibn Al Quff hospital for spinal cord injuries, Baghdad, Iraq. The data collection was done during the period from June, 1st, 2011 to November, 1st, 2012. Study Population and Sampling: All inpatients with traumatic spinal cord injury, both genders were included.

\section{Inclusion criteria}

All traumatic spinal cord injured patients with paraplegia or quadriplegia, aged $\geq 18$ years, of both sexes, and accepted to participate and have the interview.

\section{Exclusion criteria}

Severe injured patients, who cannot respond to questions, age $>18$ years, with paraplegia or quadriplegia by other non-traumatic causes like medical disorder or congenital disorders, and those with substance abuse.

\section{Data collection tools}

Basic socio-demographic variables, spinal cord injury history and history of co morbid characteristic and complications were compiled using a questionnaire filled through a direct interview. Mental status of the traumatic spinal cord injured inpatient was assessed using selfreporting questionnaires scale (SRQ-20) that was developed by the $\mathrm{WHO}$ and used in many countries. According to previous studies conducted in Iraq, the cut-off point identified used to categories "potential psychiatric cases" and more generally persons with significant psychological distress was seven [20]. Those with positive SRQ-20 results were assessed for the presence of depression using the DSM-IV criteria [9]. Those with "depression" were further assessed for the severity of depression using the Hamilton scale. It contains 17 items to be rated (HAM-17) [21].

\section{Definition of variables}

The independent variables evaluated to explain depression were socio-demographics (age, gender, marital status, level of education, occupation, smoking habits, characteristics of the disability (types, causes, duration, admission times, and rehabilitation), complications and bed sores, and comorbid condition.

\section{Statistical analysis}

SPSS version 17 used for data entry and analysis. The prevalence of depression and its $95 \%$ confidence interval was calculated. Univariate analysis using Chi square was applied to identify potential risk factors of depression.

\section{Ethical issues}

Informed consent was obtained from the patients after clarifying the objectives of the study. Names were kept anonymous and interviews were conducted with full privacy.

\section{Results}

A total of 274 spinal cord injured inpatients were approached; 255 welcomed and accepted to participate (response rate: 93\%). Paraplegics were $193(75.7 \%)$ and quadriplegics were 62 (24.3\%). Distribution by socio-demographic and spinal cord injury characteristics and comorbid conditions are shown in (Tables 1 and 2).
The prevalence of depression was $74.1 \%$. About $44.7 \%$ of the sample has severe and very severe depression. None were receiving treatment; psychotherapy or medications. A cross classification of patients with and without depression by socio-demographic and spinal cord injury patients characteristics and co-morbid conditions is shown in (Tables 3 and 4$)$.

The degree of severity of depression was explored according to socio-demographic characteristics and other co morbid features associated with spinal cord injury shown in (Tables 5 and 6).

The prevalence of depression was highest among those aged (46$55)$ years $(91.66 \%)$ and lowest among those aged $26-35$ years $(52.17 \%)$ $(\mathrm{P}=0.001)$. Females had significantly higher proportion of depression (94.12\%) compared to males $(69.12 \%)(\mathrm{P}=0.001)$. Depression was significantly higher among illiterate $(90 \%)$ than other educated patients $(\mathrm{P}=0.038)$. Unemployed (85.7\%) and housekeepers (92.2\%) patients had significantly higher prevalence of depression than employed patients $(66.6 \%)(\mathrm{P}=0.003)$. Depression was significantly higher among smokers $(83.3 \%)$ than non- smokers $(\mathrm{P}=0.035)$.

The prevalence of depression was not significantly different by marital status $(\mathrm{P}=0.258)$, types of disability $(\mathrm{P}=0.727)$, and causes of spinal cord injury $(\mathrm{P}=0.086)$. The prevalence of depression was highest among those with injury of spinal cord for 1-5years duration $(90.6 \%)$ $(\mathrm{P}=0.003)$, those with frequent admissions to the hospital $(89.3 \%)$ $(\mathrm{P}=0.001)$, those who have other comorbid illnesses $(\mathrm{P}=0.018)$, and among those exposed to life events $(\mathrm{P}=0.008)$.

The prevalence of depression was not significantly affected by family history of mental illness $(\mathrm{P}=0.116)$, duration of admission $(\mathrm{P}=0.744)$, accompanied persons $(\mathrm{P}=0.688)$, visitors $(\mathrm{P}=0.646)$, rehabilitation $(\mathrm{P}=0.434)$, walking aids $(\mathrm{P}=0.935)$, complications $(\mathrm{P}=0.253)$, and presence of bed sores $(\mathrm{P}=0.324)$.

\begin{tabular}{|c|c|c|c|c|c|}
\hline \multirow{2}{*}{\multicolumn{2}{|c|}{ Socio demographic characteristic }} & \multicolumn{2}{|c|}{ Spinal Cord Injury } & \multicolumn{2}{|c|}{ Total (255) } \\
\hline & & \multirow{2}{*}{\begin{tabular}{|c|} 
Paraplegia \\
70
\end{tabular}} & \multirow{2}{*}{$\begin{array}{c}\text { Tetraplegia } \\
23\end{array}$} & \multirow{2}{*}{$\begin{array}{c}\text { No. } \\
93\end{array}$} & \multirow{2}{*}{$\begin{array}{l}\text { (\%) } \\
36.5\end{array}$} \\
\hline \multirow{5}{*}{ Age Group } & 18 yrs - 25 yrs & & & & \\
\hline & 26 yrs - 35 yrs & 51 & 18 & 69 & 27.1 \\
\hline & 36 yrs - 45 yrs & 33 & 9 & 42 & 16.5 \\
\hline & 46 yrs - 55 yrs & 28 & 8 & 36 & 14.1 \\
\hline & 56 yrs -65 yrs & 11 & 4 & 15 & 5.9 \\
\hline \multirow{2}{*}{ Sex } & Male & 161 & 43 & 204 & 80 \\
\hline & Female & 32 & 19 & 51 & 20 \\
\hline \multirow{3}{*}{ Marital Status } & Single & 90 & 36 & 126 & 49.4 \\
\hline & Married & 98 & 25 & 123 & 48.2 \\
\hline & Divorced & 5 & 1 & 6 & 2.4 \\
\hline \multirow{5}{*}{$\begin{array}{l}\text { Education } \\
\text { Level }\end{array}$} & Illiterate & 19 & 11 & 30 & 11.8 \\
\hline & Primary & 92 & 31 & 123 & 48.2 \\
\hline & Intermediate & 50 & 13 & 63 & 24.7 \\
\hline & Secondary & 18 & 6 & 24 & 9.4 \\
\hline & Institute and college & 14 & 1 & 15 & 5.9 \\
\hline \multirow{7}{*}{ Occupation } & Unemployed & 20 & 1 & 21 & 8.2 \\
\hline & Employed & 16 & 2 & 18 & 7.1 \\
\hline & Free work & 85 & 35 & 120 & 47.1 \\
\hline & House wife & 23 & 16 & 39 & 15.3 \\
\hline & Military & 23 & 4 & 27 & 10.6 \\
\hline & Retired & 3 & 0 & 3 & 1.2 \\
\hline & Student & 23 & 4 & 27 & 10.6 \\
\hline \multirow{2}{*}{ Smoking } & No & 137 & 46 & 183 & 71.8 \\
\hline & Yes & 56 & 16 & 72 & 28.2 \\
\hline
\end{tabular}

Table 1: Distribution of the study group by socio-demographic characteristics and smoking habit. 
Citation: Al Abbudi SJR, Ezzat KI, Zebala AA, Hamdy DJ, Al-Beedany MSJ, et al. (2017) Prevalence and Determinants of Depression Among Traumatic Spinal Cord Injured Patients Attending Ibn-Al-Quff Hospital, Baghdad, Iraq. J Psychiatry 20: 428. doi:10.4172/23785756.1000428

Page 3 of 7

\begin{tabular}{|c|c|c|c|c|c|}
\hline & & \multicolumn{2}{|c|}{ Spinal Cord Injury } & \multicolumn{2}{|c|}{ Total (255) } \\
\hline & & Paraplegia & Quadriplegia & No. & $(\%)$ \\
\hline \multirow{4}{*}{ Cause of injuries } & Bullet & 28 & 12 & 40 & 15.7 \\
\hline & Shell Explosion & 48 & 41 & 89 & 34.9 \\
\hline & $\mathrm{FFH}$ & 45 & 5 & 50 & 19.6 \\
\hline & RTA & 72 & 4 & 76 & 29.8 \\
\hline \multirow{3}{*}{ Duration of Injury } & Less than 1 year & 104 & 27 & 131 & 51.4 \\
\hline & $1-5$ years & 46 & 7 & 53 & 20.8 \\
\hline & More than 5 years & 43 & 28 & 71 & 27.8 \\
\hline \multirow{3}{*}{ Duration of Admission } & Less than 1 month & 14 & 7 & 21 & 8.2 \\
\hline & 1 - 6 months & 148 & 47 & 195 & 76.5 \\
\hline & More than 6 months & 31 & 8 & 39 & 15.3 \\
\hline \multirow{2}{*}{ Times of Admission } & First Admission & 133 & 38 & 171 & 67.1 \\
\hline & Frequent Admissions & 60 & 24 & 84 & 32.9 \\
\hline \multirow{2}{*}{ Accompanied Persons } & Absent & 33 & 18 & 51 & 20 \\
\hline & Present & 160 & 44 & 204 & 80 \\
\hline \multirow{2}{*}{ Visitors } & Absent & 20 & 7 & 27 & 10.6 \\
\hline & Present & 173 & 55 & 228 & 89.4 \\
\hline \multirow{2}{*}{ Rehabilitation } & Absent & 26 & 4 & 30 & 11.8 \\
\hline & Present & 167 & 58 & 225 & 88.2 \\
\hline \multirow{2}{*}{ Walking Aids } & Absent & 42 & 13 & 55 & 21.6 \\
\hline & Present & 151 & 49 & 200 & 78.4 \\
\hline \multirow{2}{*}{ Complication } & Absent & 81 & 27 & 108 & 42.4 \\
\hline & Present & 112 & 35 & 147 & 57.6 \\
\hline \multirow{2}{*}{ Co morbidity } & Absent & 185 & 55 & 240 & 94.1 \\
\hline & Present & 8 & 7 & 15 & 5.9 \\
\hline \multirow{2}{*}{ Pressure sore } & Absent & 131 & 30 & 161 & 63.1 \\
\hline & Present & 62 & 32 & 94 & 36.9 \\
\hline
\end{tabular}

Table 2: Distribution of the study group by spinal cord injury characteristics and co-morbid conditions.

\begin{tabular}{|c|c|c|c|c|c|c|c|c|}
\hline & & \multicolumn{4}{|c|}{ Depression } & \multirow{2}{*}{\multicolumn{2}{|c|}{ Total (255) }} & \multirow{3}{*}{$P$ value } \\
\hline & & \multicolumn{2}{|c|}{ Not Depressed } & \multicolumn{2}{|c|}{ Depressed } & & & \\
\hline & & No. & $(\%)$ & No. & $(\%)$ & No & (\%) & \\
\hline \multirow{5}{*}{ Age Group } & 18 yrs - 25 yrs & 21 & 22.6 & 72 & 77.4 & 93 & 36.47 & \multirow{5}{*}{0.001} \\
\hline & 26 yrs - 35 yrs & 33 & 47.83 & 36 & 52.17 & 69 & 27.05 & \\
\hline & 36 yrs - 45 yrs & 6 & 14.29 & 36 & 85.71 & 42 & 16.47 & \\
\hline & 46 yrs - 55 yrs & 3 & 8.34 & 33 & 91.66 & 36 & 14.11 & \\
\hline & 56 yrs - 65 yrs & 3 & 20 & 12 & 80 & 15 & 5.88 & \\
\hline \multirow{2}{*}{ Sex } & Male & 63 & 30.88 & 141 & 69.12 & 204 & 80 & \multirow{2}{*}{0.001} \\
\hline & Female & 3 & 5.88 & 48 & 94.12 & 51 & 20 & \\
\hline \multirow{3}{*}{ Marital Status } & Single & 36 & 28.57 & 90 & 71.43 & 126 & 49.41 & \multirow{3}{*}{0.258} \\
\hline & Married & 30 & 24.39 & 93 & 75.61 & 123 & 48.23 & \\
\hline & Divorced & 0 & 0 & 6 & 100 & 6 & 2.35 & \\
\hline \multirow{5}{*}{ Education Level } & Illiterate & 3 & 10 & 27 & 90 & 30 & 11.76 & \multirow{5}{*}{0.038} \\
\hline & Primary & 42 & 34.14 & 81 & 65.86 & 123 & 48.23 & \\
\hline & Intermediate & 12 & 19.05 & 51 & 80.95 & 63 & 24.7 & \\
\hline & Secondary & 6 & 25 & 18 & 75 & 24 & 9.41 & \\
\hline & College+ & 3 & 20 & 12 & 80 & 15 & 5.88 & \\
\hline
\end{tabular}


Citation: Al Abbudi SJR, Ezzat KI, Zebala AA, Hamdy DJ, Al-Beedany MSJ, et al. (2017) Prevalence and Determinants of Depression Among Traumatic Spinal Cord Injured Patients Attending Ibn-Al-Quff Hospital, Baghdad, Iraq. J Psychiatry 20: 428. doi:10.4172/23785756.1000428

Page 4 of 7

\begin{tabular}{|c|c|c|c|c|c|c|c|c|}
\hline \multirow{7}{*}{ Occupation } & Unemployed & 3 & 14.28 & 18 & 85.72 & 21 & 8.23 & \multirow{7}{*}{0.003} \\
\hline & Employed & 6 & 33.33 & 12 & 66.67 & 18 & 7.05 & \\
\hline & free work & 30 & 25 & 90 & 75 & 120 & 47.05 & \\
\hline & house keeper & 3 & 7.69 & 36 & 92.2 & 39 & 15.29 & \\
\hline & Military & 12 & 44.44 & 15 & 55.56 & 27 & 10.58 & \\
\hline & Retired & 0 & 0 & 3 & 100 & 3 & 1.17 & \\
\hline & Student & 12 & 44.44 & 15 & 55.56 & 27 & 10.58 & \\
\hline \multirow{2}{*}{ Smoking } & No & 54 & 29.5 & 129 & 70.5 & 183 & 71.76 & \multirow{2}{*}{0.035} \\
\hline & Yes & 12 & 16.67 & 60 & 83.33 & 72 & 28.23 & \\
\hline \multicolumn{2}{|c|}{ Total } & 66 & 25.88 & 189 & 74.12 & 255 & $100 \%$ & \\
\hline
\end{tabular}

Table 3: Distribution of the study group by depression and the socio-demographic characteristics.

\begin{tabular}{|c|c|c|c|c|c|c|c|c|}
\hline & & \multicolumn{4}{|c|}{ Depression } & \multirow{2}{*}{\multicolumn{2}{|c|}{ Total (255) }} & \multirow{3}{*}{$P$ value } \\
\hline & & \multicolumn{2}{|c|}{ Not Depressed } & \multicolumn{2}{|c|}{ Depressed } & & & \\
\hline & & No. & $\%$ & No. & $\%$ & No. & $\%$ & \\
\hline \multirow{2}{*}{ Disability } & Paraplegia & 51 & 26.4 & 142 & 73.6 & 193 & 75.7 & \multirow{2}{*}{0.727} \\
\hline & Quadriplegia & 15 & 24.2 & 47 & 75.8 & 62 & 24.3 & \\
\hline \multirow{4}{*}{ Cause of injuries } & Bullet & 4 & 10 & 36 & 90 & 40 & 15.7 & \multirow{4}{*}{0.086} \\
\hline & Shell Explosion & 25 & 28.1 & 64 & 71.9 & 89 & 34.9 & \\
\hline & FFH & 16 & 32 & 34 & 68 & 50 & 19.6 & \\
\hline & RTA & 21 & 27.6 & 55 & 72.4 & 76 & 29.8 & \\
\hline \multirow{3}{*}{ Duration of injury } & UP TO 1 YEAR & 44 & 33.6 & 87 & 66.4 & 131 & 51.37 & \multirow{3}{*}{0.003} \\
\hline & 1 - 5 YEARS & 5 & 9.4 & 48 & 90.6 & 53 & 20.8 & \\
\hline & MORE THAN 5 YEARS & 17 & 23.9 & 54 & 76.1 & 71 & 27.8 & \\
\hline \multirow{3}{*}{ Duration of admission } & LESS THAN 1 MONTH & 5 & 23.8 & 16 & 76.2 & 21 & 8.2 & \multirow{3}{*}{.0744} \\
\hline & 1 - 6 MONTHS & 49 & 25.1 & 146 & 74.9 & 195 & 76.5 & \\
\hline & MORE THAN 6 MONTHS & 12 & 30.8 & 27 & 69.2 & 39 & 15.3 & \\
\hline \multirow{2}{*}{ Times of Admission } & First Admission & 57 & 33.3 & 114 & 66.7 & 171 & 67.1 & \multirow{2}{*}{0.000} \\
\hline & Frequent Admissions & 9 & 10.7 & 75 & 89.3 & 84 & 32.9 & \\
\hline \multirow{2}{*}{ Accompanied persons } & No & 12 & 23.5 & 39 & 76.5 & 51 & 20 & \multirow{2}{*}{0.688} \\
\hline & Yes & 54 & 26.5 & 150 & 73.5 & 204 & 80 & \\
\hline \multirow{2}{*}{ Visitors } & No & 6 & 22.2 & 21 & 77.8 & 27 & 10.6 & \multirow{2}{*}{0,646} \\
\hline & Yes & 60 & 26.3 & 168 & 73.7 & 228 & 89.4 & \\
\hline \multirow{2}{*}{ Rehabilitation } & No & 6 & 20 & 24 & 80 & 30 & 11.7 & \multirow{2}{*}{0.434} \\
\hline & Yes & 60 & 26.7 & 165 & 73.3 & 225 & 88.2 & \\
\hline \multirow{2}{*}{ walking aids } & No & 14 & 25.5 & 41 & 74.5 & 55 & 21.6 & \multirow{2}{*}{0.935} \\
\hline & Yes & 52 & 26 & 148 & 74 & 200 & 78.4 & \\
\hline \multirow{2}{*}{ Complication } & No & 24 & 22.2 & 84 & 77.8 & 108 & 42.4 & \multirow{2}{*}{0.253} \\
\hline & Yes & 42 & 28.6 & 105 & 71.4 & 147 & 57.6 & \\
\hline \multirow{2}{*}{ Bedsores } & No & 45 & 28 & 116 & 72. & 161 & 63.1 & \multirow{2}{*}{0.324} \\
\hline & Yes & 21 & 22.3 & 73 & 77.7 & 94 & 36.9 & \\
\hline \multirow[t]{2}{*}{ Co morbidity } & No & 66 & 27.5 & 174 & 72.5 & 240 & 94.1 & \multirow{2}{*}{0.018} \\
\hline & Yes & 0 & 0 & 15 & 100 & 15 & 5.9 & \\
\hline & tal & 66 & 25.88 & 189 & 74.12 & 255 & 100 & \\
\hline
\end{tabular}

Table 4: Distribution of the study group by depression and spinal cord injury characteristics and presence of complication or comorbid condition.

\begin{tabular}{|c|c|c|c|c|c|c|c|c|c|c|c|c|c|c|}
\hline & & \multicolumn{10}{|c|}{ Depression } & \multicolumn{2}{|c|}{ Total (255) } & \multirow{3}{*}{$P$ value } \\
\hline & & \multicolumn{2}{|c|}{ No Depression } & \multicolumn{2}{|c|}{$\begin{array}{c}\text { Mild } \\
\text { Depression }\end{array}$} & \multicolumn{2}{|c|}{$\begin{array}{l}\text { Moderate } \\
\text { Depression }\end{array}$} & \multicolumn{2}{|c|}{$\begin{array}{c}\text { Severe } \\
\text { Depression }\end{array}$} & \multicolumn{2}{|c|}{$\begin{array}{l}\text { Very Severe } \\
\text { Depression }\end{array}$} & \multirow[t]{2}{*}{ No. } & \multirow[t]{2}{*}{$(\%)$} & \\
\hline & & No & $\%$ & No & $\%$ & No & $\%$ & No & $\%$ & No & $\%$ & & & \\
\hline \multirow{5}{*}{ Age Group } & 18 yrs - 25 yrs & 21 & 22.5 & 3 & 3.2 & 21 & 22.5 & 18 & 19.3 & 30 & 32.2 & 93 & 36.5 & \multirow{5}{*}{0.000} \\
\hline & 26 yrs - 35 yrs & 33 & 47.8 & 6 & 8.7 & 9 & 13.05 & 9 & 13.05 & 12 & 17.4 & 69 & 27.1 & \\
\hline & 36 yrs - 45 yrs & 6 & 14.3 & 9 & 21.43 & 6 & 14.3 & 6 & 14.3 & 15 & 35.7 & 42 & 16.5 & \\
\hline & 46 yrs - 55 yrs & 3 & 8.3 & 6 & 16.7 & 9 & 25 & 3 & 8.3 & 15 & 41.7 & 36 & 14.1 & \\
\hline & 56 yrs - 65 yrs & 3 & 20 & 6 & 40 & 0 & 0 & 0 & 0 & 6 & 40 & 15 & 5.9 & \\
\hline
\end{tabular}


Citation: Al Abbudi SJR, Ezzat KI, Zebala AA, Hamdy DJ, Al-Beedany MSJ, et al. (2017) Prevalence and Determinants of Depression Among Traumatic Spinal Cord Injured Patients Attending Ibn-Al-Quff Hospital, Baghdad, Iraq. J Psychiatry 20: 428. doi:10.4172/23785756.1000428

Page 5 of 7

\begin{tabular}{|c|c|c|c|c|c|c|c|c|c|c|c|c|c|c|}
\hline \multirow{2}{*}{ Sex } & Male & 63 & 30.9 & 27 & 13.2 & 42 & 20.6 & 24 & 11.8 & 48 & 23.5 & 204 & 80 & \multirow{2}{*}{0.000} \\
\hline & Female & 3 & 5.9 & 3 & 5.9 & 3 & 5.9 & 12 & 23.5 & 30 & 58.8 & 51 & 20 & \\
\hline \multirow{3}{*}{ Marital Status } & Single & 36 & 28.6 & 9 & 7.1 & 27 & 21.4 & 18 & 14.3 & 36 & 28.6 & 126 & 49.4 & \multirow{3}{*}{0.029} \\
\hline & Married & 30 & 24.4 & 18 & 14.6 & 18 & 14.6 & 18 & 14.6 & 39 & 31.7 & 123 & 48.2 & \\
\hline & Divorced & 0 & 0 & 3 & 50 & 0 & 0 & 0 & 0 & 3 & 50 & 6 & 2.4 & \\
\hline \multirow{5}{*}{$\begin{array}{l}\text { Education } \\
\text { Level }\end{array}$} & Illiterate & 3 & 10 & 3 & 10 & 3 & 10 & 3 & 10 & 18 & 60 & 30 & 11.8 & \multirow{5}{*}{0.000} \\
\hline & Primary & 42 & 34.1 & 12 & 9.8 & 30 & 24.4 & 15 & 12.2 & 24 & 19.5 & 123 & 48.2 & \\
\hline & intermediate & 12 & 19.05 & 9 & 14.3 & 12 & 19.05 & 9 & 14.3 & 21 & 33.3 & 63 & 24.7 & \\
\hline & Secondary & 6 & 25 & 6 & 25 & 0 & 0 & 6 & 25 & 6 & 25 & 24 & 9.4 & \\
\hline & College+ & 3 & 20 & 0 & 0 & 0 & 0 & 3 & 20 & 9 & 60 & 15 & 5.9 & \\
\hline \multirow{7}{*}{ Occupation } & Unemployed & 3 & 14.3 & 0 & 0 & 0 & 0 & 6 & 28.6 & 12 & 57.14 & 21 & 8.2 & \multirow{7}{*}{0.000} \\
\hline & Employed & 6 & 33.3 & 3 & 16.7 & 0 & 0 & 0 & 0 & 9 & 50 & 18 & 7.1 & \\
\hline & Free work & 30 & 25 & 15 & 12.5 & 36 & 30 & 15 & 12.5 & 24 & 20 & 120 & 47.1 & \\
\hline & House keeper & 3 & 7.7 & 3 & 7.7 & 3 & 7.7 & 9 & 23.1 & 21 & 53.9 & 39 & 15.3 & \\
\hline & Military & 12 & 44.4 & 3 & 11.1 & 6 & 22.2 & 3 & 11.1 & 3 & 11.1 & 27 & 10.6 & \\
\hline & Retired & 0 & 0 & 3 & 100 & 0 & 0 & 0 & 0 & 0 & 0 & 3 & 1.2 & \\
\hline & Student & 12 & 44.4 & 3 & 11.1 & 0 & 0 & 3 & 11.1 & 9 & 33.3 & 27 & 10.6 & \\
\hline \multirow[t]{2}{*}{ Smoking } & Not smoker & 54 & 29.5 & 24 & 13.1 & 15 & 8.2 & 24 & 13.1 & 66 & 36.1 & 183 & 71.8 & \multirow{2}{*}{0.000} \\
\hline & Smoker & 12 & 16.7 & 6 & 8.3 & 30 & 41.6 & 12 & 16.7 & 12 & 16.7 & 72 & 28.2 & \\
\hline Total & & 66 & & 30 & & 45 & & 36 & & 78 & & 255 & $100 \%$ & \\
\hline
\end{tabular}

Table 5: Distribution of the study group by degree of severity of depression and the socio-demographic characteristics.

\begin{tabular}{|c|c|c|c|c|c|c|c|c|c|c|c|c|c|c|}
\hline & & \multicolumn{10}{|c|}{ Depression } & \multicolumn{2}{|c|}{ Total (255) } & \multirow{3}{*}{$P$ value } \\
\hline & & \multicolumn{2}{|c|}{ No Depression } & \multicolumn{2}{|c|}{ Mild Depression } & \multicolumn{2}{|c|}{$\begin{array}{l}\text { Moderate } \\
\text { Depression }\end{array}$} & \multicolumn{2}{|c|}{$\begin{array}{c}\text { Severe } \\
\text { Depression }\end{array}$} & \multicolumn{2}{|c|}{$\begin{array}{l}\text { Very Severe } \\
\text { Depression }\end{array}$} & \multirow[t]{2}{*}{ No. } & \multirow[t]{2}{*}{$\%$} & \\
\hline & & No. & $\%$ & No. & $\%$ & No. & $\%$ & No. & $\%$ & No. & $\%$ & & & \\
\hline \multirow{4}{*}{ Cause of injuries } & Bullet & 4 & 10 & 1 & 2.5 & 6 & 15 & 13 & 32.5 & 16 & 40 & 40 & 15.7 & \multirow{4}{*}{0.010} \\
\hline & Shell Explosion & 25 & 28.1 & 15 & 16.9 & 14 & 15.74 & 8 & 8.99 & 27 & 30.3 & 89 & 34.9 & \\
\hline & FFH & 16 & 32 & 5 & 10 & 10 & 20 & 3 & 6 & 16 & 32 & 50 & 19.6 & \\
\hline & RTA & 21 & 27.6 & 9 & 11.85 & 15 & 19.7 & 12 & 15.79 & 19 & 25 & 76 & 29.8 & \\
\hline \multirow{3}{*}{ Duration of injury } & Less than 1 year & 44 & 33.6 & 15 & 11.5 & 21 & 16.03 & 21 & 16.03 & 30 & 22.9 & 131 & 51.4 & \multirow{3}{*}{0.011} \\
\hline & $1-5$ years & 5 & 9.4 & 6 & 11.32 & 9 & 17 & 6 & 11.32 & 27 & 50.9 & 53 & 20.8 & \\
\hline & More than 5 years & 17 & 23.95 & 9 & 12.7 & 15 & 21.1 & 9 & 12.7 & 21 & 29.6 & 71 & 27.8 & \\
\hline \multirow{3}{*}{$\begin{array}{l}\text { Duration of } \\
\text { admission }\end{array}$} & Less than 1 month & 5 & 23.8 & 5 & 23.8 & 7 & 33.3 & 0 & 0 & 4 & 19.05 & 21 & 8.2 & \multirow{3}{*}{0.089} \\
\hline & 1 - 6 months & 49 & 25.1 & 22 & 11.3 & 32 & 16.4 & 33 & 16.9 & 59 & 30.3 & 195 & 76.5 & \\
\hline & More than 6 months & 12 & 30.8 & 3 & 7.7 & 6 & 15.4 & 3 & 7.7 & 15 & 38.5 & 39 & 15.3 & \\
\hline \multirow{2}{*}{$\begin{array}{c}\text { Times of } \\
\text { Admission }\end{array}$} & First Admission & 57 & 33.3 & 24 & 14.04 & 24 & 14.0 & 24 & 14.04 & 42 & 24.6 & 171 & 67.1 & \multirow{2}{*}{0.000} \\
\hline & Frequent Admissions & 9 & 10.7 & 6 & 7.14 & 21 & 25 & 12 & 14.3 & 36 & 42.9 & 84 & 32.9 & \\
\hline \multirow{2}{*}{$\begin{array}{l}\text { Accompanied } \\
\text { persons }\end{array}$} & Absent & 12 & 23.6 & 6 & 11.8 & 12 & 23.5 & 3 & 5.9 & 18 & 35.3 & 51 & 20 & \multirow{2}{*}{0.297} \\
\hline & Present & 54 & 26.5 & 24 & 11.8 & 33 & 16.2 & 33 & 16.2 & 60 & 29.4 & 204 & 80 & \\
\hline \multirow{2}{*}{ Visitors } & Absent & 6 & 22.2 & 3 & 11.1 & 3 & 11.1 & 6 & 22.2 & 9 & 33.3 & 27 & 10.6 & \multirow{2}{*}{0.667} \\
\hline & Present & 60 & 26.3 & 27 & 11.8 & 42 & 18.4 & 30 & 13.2 & 69 & 30.3 & 228 & 89.4 & \\
\hline \multirow{2}{*}{ Rehabilitation } & Absent & 6 & 20 & 0 & 0 & 9 & 30 & 6 & 20 & 9 & 30 & 30 & 11.8 & \multirow{2}{*}{0.083} \\
\hline & Present & 60 & 26.7 & 30 & 13.3 & 36 & 16 & 30 & 13.3 & 69 & 30.7 & 225 & 88.2 & \\
\hline \multirow{2}{*}{ walking aids } & Absent & 14 & 25.5 & 7 & 12.7 & 15 & 27.3 & 6 & 10.9 & 13 & 23.6 & 55 & 21.6 & \multirow{2}{*}{0.252} \\
\hline & Present & 52 & 26 & 23 & 11.5 & 30 & 15 & 30 & 15 & 65 & 32.5 & 200 & 78.4 & \\
\hline \multirow{2}{*}{ Complication } & Absent & 24 & 22.2 & 18 & 16.7 & 24 & 22.2 & 21 & 19.4 & 21 & 19.4 & 108 & 42.4 & 0001 \\
\hline & Present & 42 & 28.6 & 12 & 8.2 & 21 & 14.3 & 15 & 10.2 & 57 & 38.8 & 147 & 57.6 & 0.001 \\
\hline & Absent & 66 & 27.5 & 27 & 11.3 & 39 & 16.3 & 36 & 15 & 72 & 30 & 240 & 94.1 & \\
\hline Co morbidity & Present & 0 & 0 & 3 & 20 & 6 & 40 & 0 & 0 & 6 & 40 & 15 & 5.9 & 0.015 \\
\hline Bedsores & Absent & 45 & 28 & 23 & 14.3 & 23 & 14.3 & 20 & 12.4 & 50 & 31.1 & 161 & 63.1 & 0.147 \\
\hline Bedsores & Present & 21 & 22.3 & 7 & 7.45 & 22 & 23.4 & 16 & 17.02 & 28 & 29.8 & 94 & 36.9 & $0.14 t$ \\
\hline & Total & 66 & 25.9 & 30 & 11.8 & 45 & 17.65 & 36 & 14.1 & 78 & 30.6 & 255 & $100 \%$ & \\
\hline
\end{tabular}

Table 6: Distribution of the study group by degree of severity of depression and spinal cord injury characteristics and presence of complication or comorbid condition. 
Citation: Al Abbudi SJR, Ezzat KI, Zebala AA, Hamdy DJ, Al-Beedany MSJ, et al. (2017) Prevalence and Determinants of Depression Among Traumatic Spinal Cord Injured Patients Attending Ibn-Al-Quff Hospital, Baghdad, Iraq. J Psychiatry 20: 428. doi:10.4172/23785756.1000428

Page 6 of 7

The assessment of depressed spinal cord injured patients by severity of depression revealed that $25.9 \%$ had mild depression, $11.8 \%$ had moderate depression and $44.7 \%$ had severe or very severe depression (Tables 5 and 6). The severity of depression was significantly associated with socio-demographic characteristics; age of the patients $(\mathrm{P}=0.000)$, gender $(\mathrm{P}=0.000)$, marital status $(\mathrm{P}=0.029)$, education level $(\mathrm{P}=0.000)$, occupation $(\mathrm{P}=0.000)$, and smoking habit $(\mathrm{P}=0.000)$.

The severity of depression was significantly associated with the causes of spinal cord injury $(\mathrm{P}=0.010)$, duration of injury $(\mathrm{P}=0.011)$, times of admission $(\mathrm{P}=0.000)$, complications $(\mathrm{P}=0.001)$, and comorbidity $(\mathrm{P}=0.015)$.

\section{Discussion}

The prevalence of depression was $74.1 \%$ which is higher than many studies done across cultures. There was a strong correlation between degree of severity of depression and socio-demographic characteristics of the SCI inpatients. American meta-analysis (2014) of 19 studies found the mean prevalence estimate of depression diagnosis after SCI was $22.2 \%$ [22]. In a number of studies it has been reported that depression scores varies between $20-40 \%$ in SCI patients [23-25]. In Italian sample averaging 6 years post-SCI, found $16 \%$ reported significant symptoms of depression [25,26] employed an Australian sample who averaged 19 years post-SCI, 37\% were identified as depressed [26,27] study of 233 Albertans with SCI; $28.9 \%$ were treated for depression following their traumatic SCIs $[27,28]$ in a review found rates of major depression following SCI to vary widely across studies and can range from $7 \%$ to $31 \%$ of studied population $[28,29]$ surveyed 568 adult traumatic SCI inpatient rehabilitation clients; approximately $22 \%$ met self-reported symptoms consistent with major depressive disorder [28,29] surveyed 849 SCI outpatients at one-year post injury and found $11.4 \%$ met criteria for MDD [27,30] suggest a $42 \%$ overall rate of depression with a $21 \%$ probable rate of major depression [30]

Prevalence of depression of this study (74.1\%) was lower than result of study done in Bangladesh (2007) on 167 spinal cord injured patients that found rate of depression to be around $80.24 \%$ [31]. Iranian study (2004) showed that the prevalence of depression in physically disabled veterans was (71\%) [5], while recent Iranian study (2015) found 91 of $226(40.2 \%)$ had moderate to severe depression [32]. Estimates of the prevalence of depression are affected by the nature of the measures used, how depression is defined, aging characteristics of the samples studied, and when symptoms are assessed post-injury.

Current study found that depression and severity of depression among spinal cord injured patients significantly associated with duration of disability; $66.4 \%, 90.6 \%, 76.1 \%$ for duration; 1 year, $1-5$ years, $<5$ years respectively, while Richardson and Richards, in a cross sectional study, found that rates of clinically significant depressive symptoms were reported by approximately $21 \%, 18 \%, 12 \%$ and $12 \%$ of SCI survivors surveyed at $1,5,15$ and 25 years post injury, suggesting rates tended to decrease with time since injury [33,34] followed 411 SCI model system participants and found approximately $20 \%$ of at 1 year post injury and $18 \%$ at year 5 post-injury reported symptoms consistent with major depression [34,35]. In a longitudinal analysis, found a substantial relationship between reported depressive symptoms at 3 months and approximately a decade post injury, with $38 \%$ and $35 \%$ of SCI survivors surveyed meeting a criterion for moderate depression at these times [35,36] reported that anxiety, depression and hopelessness gradually increased beginning at week 30 post injury and continued until discharge from rehabilitation (week 48). At that point $60 \%$ of SCI clients scored above a clinical cut-off for depression (i.e., Beck Depression Inventory) [36]. This study showed significant gender association with depression and severity of depression, while Kalpakjian and Albright founded an absence of gender differences in probable major depression and symptom severity [37]. Turkish study that depression was more frequent in females [6].

Current study found no statistical significance between depression and patient receiving rehabilitation or not. Krause et al. suggested that depressive symptoms may not peak during inpatient rehabilitation and it may take additional time for the "low point of emotional adaptation to appear" [29].

In this study, no any patient received treatment; dedication or psychotherapy. In a review of American veterans with spinal cord injuries and disabilities, Smith et al. [38] concluded that many may not be receiving adequate treatment for depression and the authors encouraged more aggressive screening and treatment [38]. Similarly, while a substantial percentage of their SCI clinic sample reported symptoms suggestive of major depression, Kemp and Krause found that none were receiving treatment (psychotherapy or medications) [39].

About $50 \%$ of causes of Spinal cord injury were bullets and shell explosions due to the security status and violence of Iraq and ongoing explosions and terrors events were major etiological factors associated with disability. Finding consistent with Sabah [18] violence is the commonest cause of Traumatic Spinal Cord Injuries in Iraq, which affect mainly the males at their most productive age.

The severity of depression of this study was the following; $25.9 \%$ mild depression, $11.8 \%$ moderate depression and $44.7 \%$ severe or very severe depression. Pakistanian study indicate the level of depression in people with physical disability, found that out of 35 individuals; $2.86 \%$ had mild mood disturbance, $2.86 \%$ had borderline clinical depression, $42.86 \%$ were moderately depressed, and $37.14 \%$ severely depressed and $14.29 \%$ were in extreme depression [40]. Whelen SR et al. found that $41 \%$ of the women with spinal cord injury had depressive symptomatology in the mild to severe range. Nearly a third of the women had very severe depressive symptomatology [12].

\section{Conclusion}

In conclusion the prevalence of depression among SCI inpatients was $74.1 \%$. About $45 \%$ of them were severely depressed. Severity of depression was significantly associated with sociodemographic characteristics, duration of disability, causes of injury, complications, and comorbidity. None of the depressed SCI patients received psychotherapy or medication. Violence was the commonest cause of Traumatic Spinal Cord Injuries. The results were compared with other studies from different cultures; prevalence of depressed spinal cord injured patients was higher than many studies and less than few.

\section{Acknowledgements}

We greatly appreciate the patients and medical staff of the Ibn Al Quff hospital for their participation and assistance with this research study.

\section{Financial Support/Disclosures}

Authors explore there was no fund for this study.

\section{Conflicts of Interest}

The authors declare no conflicts of interest.

\section{References}

1. Arango JC, Ketchum JM, Starkweather A, Nicholls E, Amber R (2011) Wilk factors predicting depression among persons with spinal cord injury 1 to 5 years post injury, NeuroRehabilitation 29: 9-21. 
Citation: Al Abbudi SJR, Ezzat KI, Zebala AA, Hamdy DJ, Al-Beedany MSJ, et al. (2017) Prevalence and Determinants of Depression Among Traumatic Spinal Cord Injured Patients Attending Ibn-Al-Quff Hospital, Baghdad, Iraq. J Psychiatry 20: 428. doi:10.4172/23785756.1000428

2. McDonald JW, Sadowsky C (2002) Spinal-cord injury. The Lancet 359: 417425.

3. Craig A, Tran Y, Middleton J (2009) Psychological morbidity and spinal cord injury: A systematic review. Spinal Cord 47: 108-114.

4. Kaplan HI, Sadok BJ (2003) Anxiety disorder chapter in synopsis of psychiatry. Kathleen Courtney Millet. Baltimore Maryland USA, pp: 623-632.

5. Vafaee B, Seidy A (2004) Prevalence of depression among physically- disabled veterans in Northwestern Iran. Iran J Med Sci 29: 43-44.

6. Koca I, Ucar M, Unal A, Tutoglu A, Boyaci A, et al. (2014) Anxiety and depression level and related factors in patients with spinal cord injury. Acta Medica Mediterranea 30: 291

7. Kennedy P, Lude P, Elfström ML, Smithson E (2010) Cognitive appraisals coping and quality of life outcome: a multi-centre study of spinal cord injury rehabilitation. Spinal Cord 48: 762-769.

8. Kennedy P, Lude P, Elfstrom ML, Smithson E (2011) Psychological contributions to functional independence: a longitudinal investigation of spinal cord injury rehabilitation. Arch Phys Med Rehabil 92: 597-602.

9. American Psychiatric Association (1994) Diagnostic and statistical manual of mental disorders (4th edn.), American Psychiatric Association, Washington, DC.

10. Kalpakjian CZ, Bombardier CH, Schomer K, Brown PA, Johnson KL (2009) Measuring depression in persons with spinal cord injury: A systematic review. J Spinal Cord Med 32: 6-24.

11. Zimmerman M, Chelminski I, McGlinchey JB, Young D (2006) Diagnosing major depressive disorder VI: Performance of an objective test as a diagnostic criterion. J Nerv Ment Dis 194: 565-569.

12. Whelen SR, Taylor HB, Hughes RB, Wenzel L, Nosek MA (2014) Depression and depression treatment in women with spinal cord injury. Top Spinal Cord Inj Rehabil 20: 23-31.

13. Fann JR, Bombardier CH, Richards JS, Tate DG, Wilson CS, et al. (2011) Depression after spinal cord injury: Comorbidities, mental health service use, and adequacy of treatment. Arch Phys Med Rehabil 92: 352-360.

14. Post MW, van Leeuwen CM (2012) Psychosocial issues in spinal cord injury: A review. Spinal Cord 50: 382-389.

15. Hoffman JM, Bombardier CH, Graves DE, Kalpakjian CZ, Krause JS (2011) A longitudinal study of depression from 1 to 5 years after spinal cord injury. Arch Phys Med Rehabil 92: 411-428.

16. Beder AR (2006) Nurses knowledge and practice concerning neurogenic bladder rehabilitation.

17. Mohamed S (2009) Effectiveness of educational program on nurses knowledge toward skin care and prevention of pressure ulcer for spinal cord injury persons.

18. Al-Rubaye SG (2012) Epidemiology and rehabilitation outcomes of traumatic spinal cord injury in Iraq.

19. AbedAli DK, Tawfiq NB (2013) Assessment of spinal cord injured persons, quality of life. Kufa Journal for Nursing Sciences 3: 231-243.

20. http://www.who.int/about/regions/emro/en/

21. Hamilton M (1960) A rating scale for depression. J Neurol Neurosurg Psychiatry 23: $56-62$.

22. Williams R, Murray A (2015) Prevalence of depression after spinal cord injury:
A meta-analysis. Arch Phys Med Rehabil 96: 133-140.

23. Craig AR, Hancock KM, Dickson HG (1994) A longitudinal investigation in to anxiety and depression over the first two years of spinal cord injury. Paraplegia 32: $675-679$

24. Shin JC, Goo HR, Yu SJ, Kim DH, Yoon SY (2012) Depression and quality of life in patients within the first 6 months after the spinal cord injury. Ann Rehabil Med 36: 119-25.

25. Scivoletto G, Petrelli A, DiLucente L, Castellano V (1997) Psychological Investigation of spinal cord patients. Spinal Cord 35: 516-520.

26. Migliorini C, Tonge B, Taleporos G (2008) Spinal cord injury and mental health. Aust N Z J Psychiatry 42: 309-314.

27. Dryden DM, Saunders LD, Rowe BH, May LA, Yiannakoulias N et al. (2004) Utilization of health services following spinal cord injury: a 6-year follow-up study. Spinal Cord 42: 513-525.

28. Bombardier CH, Richards JS, Krause JS, Tulsky D, Tate DG (2004) Symptoms of major depression in people with spinal cord injury: implications for screening. Arch Phys Med Rehabil 85: 1749-1756.

29. Krause JS, Carter RE, Pickelsimer EE, Wilson D (2008) A prospective study of health and risk of mortality after spinal cord injury. Archives of Physical Medicine \& Rehabilitation 89: 1482-1491.

30. Krause JS, Kemp B, Coker J (2000) Depression after spinal cord injury: relation to gender, ethnicity, aging, and socioeconomic indicators. Arch Phys Med Rehabil 81: 1099-1109.

31. Ahmed AU, Alam SMM, Kairy RR (2007) Need assessment of psychologically hurt young spinal injured patients in National Institute Of Traumatology \& Orthopedic Rehabilitation (NITOR) Dhaka, Bangladesh. Medical Journal 36: $39-41$

32. Mohammadi K, Rahnama P, Montazeri A (2015) Prevalence and risk factors for depression in women with multiple sclerosis: A study from Iran. Ann Gen Psychiatry 14: 29.

33. Richardson EJ, Richards JS (2008) Factor structure of the PHQ-9 screen fo depression across time since injury among persons with spinal cord injury. Rehabil Psychol 53: 243-249.

34. Hoffman JM, Bombardier CH, Graves DE, Kalpakjian CZ, Krause JS (2008) Natural history of major depression after spinal cord injury. J Spinal Cord Med 31: 236 .

35. Pollard C, Kennedy P (2007) A longitudinal analysis of emotional impact, coping strategies and post-traumatic psychological growth following spinal cord injury: A 10-year review. Br J Health Psychol 12: 347-362.

36. Kennedy P, Rogers B (2000) Anxiety and depression after spinal cord injury: A longitudinal analysis. Arch Phys Med Rehabil 81: 932-937.

37. Kalpakjian CZ, Albright KJ (2006) An examination of depression through the lens of spinal cord injury. Comparative prevalence rates and severity in women and men. Womens Health Issues 16: 380-388.

38. Smith BM, Weaver FM, Ullrich PM (2007) Prevalence of depression diagnoses and use of antidepressant medications by veterans with spinal cord injury. Am J Phys Med Rehabil 86: 662-671.

39. Kemp BJ, Krause JS (1999) Depression and life satisfaction among people ageing with post-polio and spinal cord injury. Disabil Rehabil 21: 241-249.

40. Hussain N, Sikander M, Maqsud M (2014) Level of depression in physically disabled. JRCRS 2: 12-15 